\title{
Natural killer cell activity level in colorectal cancer screening in an average risk population
}

Jandos Amankulov1,2, Dilyara Kaidarova3 , Zhamilya Zholdybay², Marianna Zagurovskaya4, Oxana Shatkovskaya ${ }^{3}$, Akmaral Ainakulova ${ }^{1}$, Madina Orazgaliyeva ${ }^{5}$

\author{
${ }^{1}$ Department of Radiology and Nuclear Medicine, Kazakh Institute of Oncology \\ and Radiology, Almaty, Kazakhstan \\ 2Department of Visual Diagnostics, Asfendiyarov Kazakh National Medical University, \\ Almaty, Kazakhstan \\ ${ }^{3}$ Department of Medical Oncology, Kazakh Institute of Oncology and Radiology, \\ Almaty, Kazakhstan \\ ${ }^{4}$ Department of Radiology, Medical College at the University of Kentucky, Lexington, \\ Kentucky, United States \\ ${ }^{5}$ Department of Genetics, Kazakh Institute of Oncology and Radiology, Almaty, \\ Kazakhstan
}

Submitted: 2 March 2021; Accepted: 9 September 2021

Online publication: 17 September 2021

Arch Med Sci

DOI: https://doi.org/10.5114/aoms/142101

Copyright (c) 2021 Termedia \& Banach

\section{Abstract}

Introduction: Increased natural killer cell activity (NKCA) is linked to reduced risk of colorectal cancer (CRC). Several prior studies have investigated the association of NKCA and the incidence of CRC in high-risk subjects. The aim of our study was to investigate NKCA sensitivity in diagnosing advanced neoplasia (AN) and CRC in an average risk population.

Material and methods: NKCA was assessed by an enzyme-linked immunosorbent assay (ELISA) blood test in average risk subjects with a range of $25-2500 \mathrm{pg} / \mathrm{ml}$ set for ELISA. NKCA higher than $200 \mathrm{pg} / \mathrm{ml}$ was defined as negative. The performance of NKCA was evaluated using measures such as sensitivity, specificity, negative and positive predictive values (NPV, PPV), clinical utility index, etc. In addition, odds ratios for developing CRC using logistic regression models were calculated.

Results: NKCA was evaluated in 354 average risk individuals (mean age: 58.5 years; $36.2 \%$ male). The diagnostic accuracy of NKCA for CRC and AN was $75.5 \%$ and $72.3 \%$ respectively, with $96.4 \%$ NPV. The NKCA test demonstrated a good negative clinical utility index for CRC and AN (0.664 and 0.741 , respectively). Individuals with low NKCA had 6.84 times higher odds of having CRC (95\% Cl: 2.31-20.27; $p<0.001)$. NKCA was higher in men vs. women $(548.5 \mathrm{pg} / \mathrm{ml}$ vs. $500.0 \mathrm{pg} / \mathrm{ml})$ and lower in smokers $(412 \mathrm{pg} / \mathrm{ml}$ vs. $544 \mathrm{pg} / \mathrm{ml}$ ), non-exercisers (413 pg/ml vs. $653.5 \mathrm{pg} / \mathrm{ml}$ ), alcohol users (389 $\mathrm{pg} / \mathrm{ml}$ vs. $476 \mathrm{pg} / \mathrm{ml}$ ), and native Kazakhs and other Asian ethnic groups (446 pg/ml vs. $514 \mathrm{pg} / \mathrm{ml}$ ).

Conclusions: A high NKCA level has potential ability to rule out CRC and AN in an average risk population.

Key words: colorectal cancer, screening, advanced adenoma, natural killer cells.

\section{Introduction}

Natural killer (NK) cells are well known for their cytolytic function within the innate immune system and act as a natural defender pro-

\author{
Corresponding author: \\ Dr. Jandos Amankulov \\ Department of Radiology \\ and Nuclear Medicine \\ Kazakh Institute \\ of Oncology and \\ Radiology \\ Department of Visual \\ Diagnostics \\ Asfendiyarov Kazakh \\ National Medical \\ University \\ Almaty, Kazakhstan \\ Phone: +77013514213 \\ E-mail: zhandos.amankulov@ \\ gmail.com
}


tecting the human organism from infection and malignant cells $[1,2]$. Several inhibitory receptors expressed on the surface of NK cells enable them to differentiate between normal and affected cells $[3,4]$ and increase their cytotoxicity against targets $[5,6]$, allowing for their efficacy in controlling cancer growth and spread.

NK cell activity (NKCA) is linked to the risk of cancer. A low level of NKCA leads to increased risk of developing cancer $[7,8]$, whereas high NKCA in the blood is associated with reduced cancer risk [9]. In addition, it has been shown that NK cells can be used as a prognostic marker in various carcinomas [10, 11]. Moreover, some studies have demonstrated a strong correlation between high NK cell cytotoxicity and reduced risk of colorectal cancer (CRC) in high risk individuals [11, 12]. At the same time, Jung et al. observed a gradual decrease in NKCA level as colonic adenoma transforms into CRC [13]. Similarly, 10 times higher risk of detecting CRC is reported with NKCA lower than $200 \mathrm{pg} / \mathrm{ml}$ [14].

While existing data on the link of NKCA and colonic neoplasia and CRC are growing in high-risk individuals, there are no data on the use of NKCA for CRC screening in the population at average risk.

Thus, the main goal of our study was to evaluate NKCA as a diagnostic marker for CRC and advanced neoplasia (AN) in an average risk population undergoing CRC screening by computed tomographic colonography (CTC). Also, the function of NK cells in association with demographic factors and health status of the general population was also assessed.

\section{Material and methods}

\section{Study population}

Average risk individuals of $45-75$ years old eligible for CRC screening were invited to participate in an NKCA test trial along with evaluation by CTC. We invited subjects who had completed both CTC screening and follow-up endoscopic colonoscopy within our previous research study [15]. Using a pre-test questionnaire, the exclusion criteria in cluded (i) any recent acute inflammatory process within 4 weeks or chronic diseases such as human immunodeficiency virus, hepatitis B or C viruses, (ii) history or current or previous malignant diseases, (iii) use of medications that may affect NK cell function, for example, immunosuppressive drugs, therapeutic immunoglobulins, methylprednisolone, anticoagulant drugs, etc. [16-18]. In addition, the average risk of participants for CRC was assured prior to the enrollment. The population at average risk for CRC comprised individuals without previously defined risk factors such as personal/family history of colorectal neoplasia and inflammatory bowel disease, having inherited syndromes linked with CRC or having type II diabetes, and others.

\section{Study design}

This prospective, cross-sectional observational study was approved by the institutional research review board and conducted at our tertiary cancer center, the Kazakh Institute of Oncology and Radiology (Almaty, Kazakhstan). All the participants have signed an informed consent form.

\section{Blood sample collection and flow cytometry assay}

On the same day as the scheduled CTC, $1 \mathrm{ml}$ of venous blood sample was collected into the NK Vue tube (NKMax, Seongnam-si, South Korea) using a standard antecubital approach. Determination of NK cell activity was performed according to the previously described established method [19]. Briefly, during the test, the whole blood sample is stimulated with cytokine that results in NK cells secreting interferon $\gamma$ (IFNG), subsequently quantified by the enzyme-linked immunosorbent assay (ELISA). A range of $25-2500 \mathrm{pg} / \mathrm{ml}$ was set for the NKCA level.

\section{Computed tomography colonoscopy (CTC), endoscopic colonoscopy (EC), and histopathology}

Scanning parameters and the protocol of CTC were according to the previously published articles [20]. Briefly, low-dose protocol (collimation $32 \times 0.6$, pitch 1.4, rotation time $0.5 \mathrm{~s}, 120 \mathrm{kVp}$, $50 \mathrm{mAs}$ ) was used for CTC acquisition. Colonic distension with carbon dioxide was achieved by an automatic insufflator. CTC examinations was evaluated by two experienced radiologists with more than 200 CTC examinations reads. CTC results were classified according to the $\mathrm{CT}$ colonography reporting and data system (C-RADS) categories [21]. C1 category of C-RADS represented a normal colon and/or benign colorectal findings; C2-C4 categories included findings consistent with colonic AN/CRC and thus considered as a positive result. All positive cases by CTC were referred for EC and subsequent biopsy and/or polypectomy. EC and histopathology evaluation were performed in accordance with the previously published standard methods [22] by endoscopists with 6 and 11 years of experience and pathologists with more than 10 years of professional experience, respectively. According to the histopathology results, colorectal lesions were classified as carcinoma, serrated or adenomatous polyps. The latter were subclassified as tubular, tubulovillous, or villous based on architectural patterns of the specimens. 
Adenomas greater than $10 \mathrm{~mm}$ and/or containing $25 \%$ and more villous component and/or those with severe dysplasia were defined as advanced adenomas. Pathologists were blinded to CTC and EC results and the CTC results were concealed from the endoscopists.

\section{Data analyses}

The qualitative values of the commercial NKCA ELISA based test were evaluated for advanced colonic adenoma and CRC. The performance of the NKCA test was evaluated using the main statistical measures such as sensitivity, specificity, negative and positive predictive values, likelihood ratio, overall test accuracy, clinical utility index, and others. To define the optimal cut-off value of NKCA a receiver operating characteristic (ROC) curve was plotted. The maximum vertical distance between the $45^{\circ}$ diagonal no chance line and the ROC curve (maximum value of Youden's index) was referred for the optimal cut-off point. Pearson correlation analysis was used to determine the association between NK cell activity and socio-demographic characteristics, and health conditions of participants. We calculated odds ratios (ORs) for developing CRC with 95\% confidence intervals (Cls) using conditional logistic regression models. We used a web-based calculator (https://www. psycho-oncology.info/cui.html) to calculate the clinical utility index (CUI). Results were considered statistically significant when $p$-values were less than 0.05. All statistical analyses were done using the SPSS software version 21.0.

\section{Results}

A total of 354 asymptomatic average risk individuals undergoing CTC screening in the city of Almaty (Kazakhstan) were enrolled in our study between June 2016 and July 2019 (Figure 1). The number of female participants was higher than males by $76.6 \%$ (226 vs. 128). Gender non-specific mean age was 58.5 years old, with male participants only slightly older than females (59.1 vs. 58.2 years old respectively). Table I provides the details on demographic and health status characteristics of the included cohort.

CTC, EC, and histological examination detected 10 cases of CRC and 32 cases of AN. In addition, 6 cases of acute/subacute colitis were detected. According to both colonoscopy methods and histology results participants were subdivided into four groups: (1) control, (2) advanced colonic adenoma, (3) active colitis (AC), and (4) CRC.

A weak linear negative relationship was found between age and level of NKCA for males and for all participants in the control group $(r=-0.232$ and $r=-0.185$, respectively, $p<0.05)$. In wom- en, there was a weak positive linear relationship between age and NKCA level, but the result was statistically non-significant $(p=0.84)$.

For the qualitative assessment, the median level of NKCA was $548.5 \mathrm{pg} / \mathrm{ml}$ for men and $500.0 \mathrm{pg} / \mathrm{ml}$ for women in the control group. The median NKCA was higher in non-smokers (544 pg/ml vs. $412 \mathrm{pg} / \mathrm{ml}$ in smokers; $p=0.03$ ) and in physically active participants $(653.5 \mathrm{pg} / \mathrm{ml}$ vs. $413 \mathrm{pg} / \mathrm{ml}$ in physically non-active; $p=0.02$ ). The

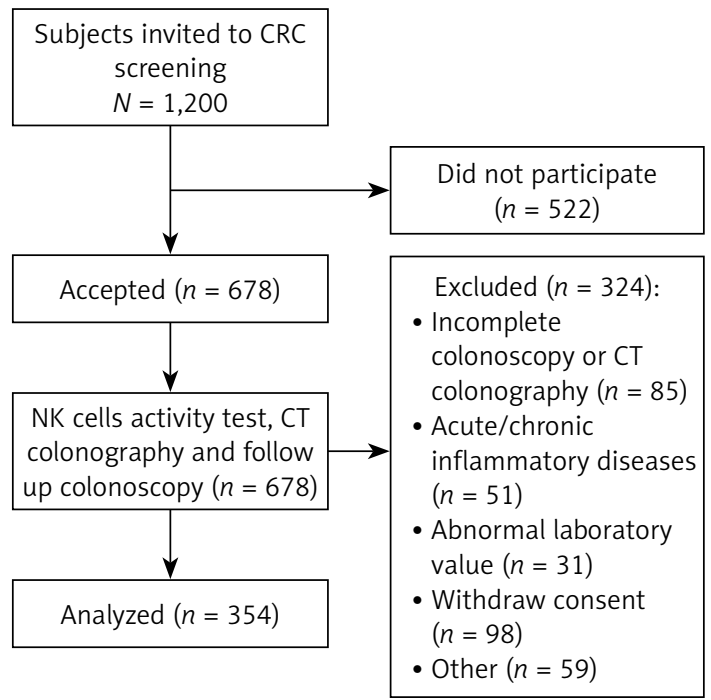

Figure 1. Flow chart of recruitment of study participants

Table I. Characteristics of participants $(n=354)$

\begin{tabular}{|lcc|}
\hline Groups & $\boldsymbol{N}$ & $\%$ \\
\hline Age [years]: & & \\
\hline $45-55$ & 135 & 38.1 \\
\hline $56-65$ & 124 & 35.0 \\
\hline $66-75$ & 95 & 26.8 \\
\hline Sex: & & \\
\hline Male & 128 & 36.2 \\
\hline Female & 226 & 63.8 \\
\hline Ethnicity: & & \\
\hline Native Kazakhs and other Asians & 252 & 71.2 \\
\hline Non-Kazakh non-Asian & 102 & 28.8 \\
\hline Physical activity [h/week] & & \\
\hline 0 & 250 & 70.6 \\
\hline$\geq 5$ & 104 & 29.4 \\
\hline Risk factors other than genetic/familial: & & \\
\hline None & 265 & 74.9 \\
\hline Tobacco smoking & 60 & 16.9 \\
\hline Alcohol consumption & 29 & 8.2 \\
\hline
\end{tabular}

$C R C$ - colorectal cancer, $N$ - number. 
alcohol abusers had an NKCA level of $389 \mathrm{pg} / \mathrm{ml}$, while the level for non-drinkers was $476 \mathrm{pg} / \mathrm{ml}$ $(p=0.12)$. NKCA level was higher in non-Asian participants compared to the Asian cohort including native Kazakhs, 514 pg/ml vs. 446 pg/ml ( $p=0.04)$.

As shown in Table II, individuals with low NKCA were 6.84 times more likely to be diagnosed with CRC than those with a higher NKCA level $(p<0.001)$. Most significant differences of CRC prevalence in association with NKCA were present in females, 22.92 times the odds of having CRC vs. males. Thus, the difference was statistically significant only for females $(p=0.004)$.

There was no statistically significant association of NKCA level and the prevalence of AN (OR = 0.62 ; $95 \% \mathrm{Cl}: 0.25-1.57, p=0.314$ ).

The median level of NKCA in CRC patients was $140 \mathrm{pg} / \mathrm{ml}$ (mean \pm SD: $171 \pm 147$ ). Patients with $\mathrm{AC}$ also had a decreased level of NKCA, $234 \mathrm{pg} / \mathrm{ml}$ (mean \pm SD: $515 \pm 732$ ). NKCA level in subjects with colonic AN was $392 \mathrm{pg} / \mathrm{ml}$ (mean \pm SD: 425 \pm 257 ). In the control group, including $C 1$ category by C-RADS, NKCA was higher in comparison with other groups, median $545 \mathrm{pg} / \mathrm{ml}$ (mean \pm SD: 679 \pm 613 ). The difference of NK cell activity level between these four groups was significant, $p<0.05$ (Table III).

The calculation of Youden's index based on ROC analysis determined the optimal NKCA cut-off value. In our study, the maximum Youden's index (0.693) was achieved at the level of $200 \mathrm{pg} / \mathrm{ml}$. The false negative rate of the test in the diagnosis of CRC at this cut-off point was $13 \%$. The level of $200 \mathrm{pg} / \mathrm{ml}$ has also been validated from the previous trial as the optimal cut-off level [14].

Diagnostic power of the NKCA test is summarized in Table IV. Overall, the sensitivity of the test for CRC was significantly higher than that for AN,

Table II. CRC odds ratio according to NK cell activity $(n=354)$

\begin{tabular}{|c|c|c|c|c|c|c|}
\hline \multirow[t]{2}{*}{ Gender } & \multicolumn{2}{|c|}{ NK cell activity $\leq 199 \mathrm{pg} / \mathrm{ml}$} & \multirow[t]{2}{*}{$P$-value } & \multicolumn{2}{|c|}{ NK cell activity $\geq 200 \mathrm{pg} / \mathrm{ml}$} & \multirow[t]{2}{*}{$P$-value } \\
\hline & $N$ & OR for CRC $(95 \% \mathrm{Cl})$ & & $N$ & OR for CRC $(95 \% \mathrm{Cl})$ & \\
\hline Men & 35 & $2.87(0.68-12.18)$ & 0.15 & 93 & $0.35(0.08-1.48)$ & 0.15 \\
\hline Women & 58 & $22.92(2.76-190.7)$ & 0.004 & 168 & $0.04(0.01-0.36)$ & 0.21 \\
\hline Both & 93 & $6.84(2.31-20.27)$ & $<0.001$ & 261 & $0.15(0.05-0.43)$ & 0.06 \\
\hline
\end{tabular}

$\mathrm{Cl}$ - confidence interval, $C R C$ - colorectal cancer, $N$ - number, $O R$ - odds ratio.

Table III. NK cell activity level in participants with CRC, AC, AN and controls $(n=354)$

\begin{tabular}{|c|c|c|c|c|c|c|c|}
\hline Groups & $N(\%)$ & Min. & Median & Max. & Mean & SD & $P$-value \\
\hline Control & $306(86.5)$ & 5.9 & 544.8 & 2516 & 679.3 & 613.0 & \\
\hline AN & $32(9.0)$ & 6.0 & 392.0 & 1009.7 & 424.8 & 257.3 & 0.04 \\
\hline$A C$ & $6(1.7)$ & 75.5 & 234.1 & 572.7 & 515.1 & 731.9 & 0.14 \\
\hline CRC & $10(2.8)$ & 25.5 & 140.4 & 422 & 171.0 & 147.4 & 0.048 \\
\hline
\end{tabular}

$A C$ - active colitis, AN - advanced neoplasia, CRC - colorectal cancer, Max. - maximum value, Min. - minimum value, $N$ - number, $S D$ - standard deviation.

Table IV. Diagnostic accuracy of NKCA in diagnosing colonic AN and CRC with the cut-off value of the test $200 \mathrm{pg} / \mathrm{ml}$ $(n=354)$

\begin{tabular}{|c|c|c|c|c|c|c|}
\hline \multirow[t]{2}{*}{ Parameter } & \multicolumn{2}{|c|}{ CRC } & \multicolumn{2}{|c|}{ AN } & \multicolumn{2}{|c|}{ CRC and AN } \\
\hline & Value & $95 \% \mathrm{Cl}$ & Value & $95 \% \mathrm{Cl}$ & Value & $95 \% \mathrm{Cl}$ \\
\hline Sensitivity & $71.4 \%$ & $41.9-91.6$ & $20.7 \%$ & $8.0-39.7$ & $35.7 \%$ & $21.6-52.0$ \\
\hline Specificity & $75.6 \%$ & $70.7-80.1$ & $73.2 \%$ & $68.1-78.0$ & $75.1 \%$ & $69.9-79.8$ \\
\hline Positive LR & 2.9 & $2.0-4.3$ & 1.1 & $0.4-1.6$ & 1.4 & $0.9-2,3$ \\
\hline Negative LR & 0.4 & $0.2-0.9$ & 1.1 & $0.9-1.3$ & 0.9 & $0.7-1.1$ \\
\hline PPV & $7.8 \%$ & $5.5-11.0$ & $1.3 \%$ & $0.6-2.7$ & $5.91 \%$ & $3.9-9.0$ \\
\hline NPV & $98.9 \%$ & $97.6-99.5$ & $98.2 \%$ & $97.8-98.5$ & $96.4 \%$ & $95.5-97.1$ \\
\hline Accuracy & $75.5 \%$ & 70.7-79.9 & $72.3 \%$ & $67.4-76.9$ & $73.4 \%$ & $68.5-78.0$ \\
\hline Prevalence & \multicolumn{2}{|c|}{$2.8 \%$} & \multicolumn{2}{|c|}{$9.0 \%$} & \multicolumn{2}{|c|}{$11.9 \%$} \\
\hline
\end{tabular}

$A N$ - advanced neoplasia, Cl - confidence interval, CRC - colorectal carcinoma, $L R$ - likelihood ratio, NPV - negative predictive value, PPV - positive predictive value. 
as well as for the combined CRC and AN group (71.4\% vs. $20.7 \%$ and $35.7 \%$, respectively). Accuracy of the NKCA test was $75.5 \%$ to detect CRC, with sensitivity of $71.4 \%$ and specificity of $75.6 \%$. The accuracy of the test in diagnosing AN was $72.3 \%$, with lower sensitivity compared to that for CRC, but relatively similar specificity $(20.7 \%$ and $73.2 \%$, respectively). Negative predictive value (NPV) of the NKCA test was high for all CRC, AN and combined CRC/AN groups (98.9\%, 98.2 and $96.4 \%$ respectively).

The NKCA test demonstrated a good utility for ruling out AN and CRC with a negative CUI of 0.741 (95\% Cl: $0.697-0.774)$ and $0.664(95 \% \mathrm{Cl}$ : 0.626-0.702), respectively, while the positive CUI was very poor $-0.053(95 \% \mathrm{Cl}: 0.018-0.096)$ vs. 0.011 (95\% Cl: 0-0.110) for AN and CRC respectively. It should also be noted that the probability that subjects with a low NKCA level truly have CRC or AN is very low (PPV $-7.8 \%$ and $1.3 \%$, respectively).

\section{Discussion}

To the best of our knowledge, our study was the first to evaluate the association of NKCA level with prevalence of $C R C$ and $A N$ in an average risk population undergoing CTC screening. The NKCA test showed good accuracy to detect CRC and AN; however, the sensitivity and specificity were lower than previously published results [14]. Specifically, in the Canadian trial, which included subjects with high risk for CRC, the NKCA test had $87.0 \%$ sensitivity and $60.8 \%$ specificity in diagnosing CRC, with PPV and NPV of $5.7 \%$ and $99.4 \%$, respectively.

Low sensitivity of the test to detect AN and very poor positive CUI values for both $C R C$ and $A N$ limit the clinical utility of the NKCA test as a primary screening test for CRC and AN. However, high NPV of the test in both conditions in our study suggests its potential ability to rule out the disease, even with an average risk for CRC.

Our study demonstrated that the likelihood of CRC is very low in average risk population, if NKCA is above $200 \mathrm{pg} / \mathrm{ml}$. At the same time, the risk of having malignant colonic neoplasms is 6.84 times higher in participants with NKCA below 200 pg/ml. Previously, it has been shown that subjects with low NKCA were at 10 times higher risk of being diagnosed with CRC than those who had values greater than $200 \mathrm{pg} / \mathrm{ml}$ [14]. But then again, the included subjects in that study were different and not entirely comparable.

The present study demonstrated that CRC patients had decreased IFNG secretion by NK cells. Tallerico et al. observed that differentiated CRC cells were highly resistant to NK cells [23]. In the previous study it was found that CRC tissues had low density or no infiltrating NK cells when the cancer tissue was evaluated immunohistochemically [24]. However, the authors found increased density of infiltrating NK cells in colonic adenoma tissue than in surrounding normal mucosa. Our findings revealed that participants with AN had a lower median NKCA level than did healthy controls. Therefore, we propose that colonic adenomas do not fully escape from NK cell-mediated immunosurveillance, as the NK cells can still migrate to adenoma tissue, as the study of Halama et al. demonstrated [24], but the function of the cells are obviously impaired. However, to date, there are no data to conclude that high serum NKCA level or high density of infiltrating NK cells in adenoma tissue can prevent adenomas from transforming into CRC or slow that process down. Therefore, analyzing the impact of NKCA on colonic AN is important in order to find ways of creating a cancer-protective environment and to develop anti-cancer drugs.

In addition, our results support healthy habits in reducing risk of colonic neoplasia, even in individuals at average risk for CRC. We have found that exercisers had a higher NKCA level than physically inactive participants. These results are congruent with previously established data on increased anti-cancer immunity with regular physical activity [25-27]. We also found that NK cell activity was higher in non-smokers than smokers. As for the alcohol consumption, the median level of NKCA was lower in this group than in smokers and physically inactive participants. These facts deserve special attention as they suggest that smoking and excessive alcohol use can cause reduced immunity, and, consequently, increased risk of cancer. Conversely, increased NKCA might have a protective effect against malignant cells. Recently, Legaz et al. ascertained that the presence of killer cell immunoglobulin-like receptors expressed on the surface of NK cells has a protective effect against alcoholic cirrhosis, which is the main risk factor for hepatocellular carcinoma [28]. However, these hypotheses must be supported by further research.

Our findings further showed that gender and ethnicity might be linked to the risk of CRC, given lower NKCA in female participants and those of Slavic descent. This is similar to the previous epidemiological study demonstrating 4 times higher prevalence of CRC in Slavic ethnic residents of Kazakhstan compared to the Asian population [29].

Several limitations of our study must be noted. The main limitation was a small sample size. Also, we used questionnaires to exclude active infections and other health conditions that can affect NKCA, but no laboratory testing was used for confirmation. In addition, the prevalence of CRC and AN was low in our cohort, which is expected given the average risk for the included individuals. This also might be a reason why PPV of the test was 
low in our study. Finally, we observed a decreasing NKCA level with increasing age, suggesting that individuals outside the $45-75$ age group may have different levels of NKCA. Thus, these differences may limit the generalizability of the study to populations of other age groups.

In conclusion, our results suggest that the NKCA test can be used as a potential biomarker to identify subjects at high risk for CRC among the general population. In addition, high level of NK cell function, regular exercise, lack of smoking and alcohol consumption habits might be linked to a lower risk of CRC detection in average risk population.

\section{Conflict of interest}

The authors declare no conflict of interest.

\section{References}

1. Baginska J, Viry E, Paggetti J, et al. The critical role of the tumor microenvironment in shaping natural killer cell-mediated anti-tumor immunity. Front Immunol 2013; 4: 490.

2. Poniewierska-Baran A, Tokarz-Deptuła B, Deptuła W. The role of innate lymphoid cells in selected disease states - cancer formation, metabolic disorder and inflammation. Arch Med Sci 2021; 17: 196-206.

3. Bessoles S, Grandclément C, Alari-Pahissa E, Gehrig J, Jeevan-Raj B, Held W. Adaptations of natural killer cells to self-MHC class I. Front Immunol 2014; 5: 349.

4. Gill S, Olson JA, Negrin RS. Natural killer cells in allogeneic transplantation: effect on engraftment, graft-versus-tumor, and graft-versus-host responses. Biol Blood Marrow Transplant 2009; 15: 765-76.

5. Tu MM, Mahmoud AB, Makrigiannis AP. Licensed and unlicensed NK cells: differential roles in cancer and viral control. Front Immunol 2016; 7: 166.

6. Thomas LM, Peterson ME, Long EO. Cutting edge: NK cell licensing modulates adhesion to target cells. J Immunol 2013; 191: 3981-5.

7. Vidal AC, Howard LE, Wiggins E, et al. Natural killer cell activity and prostate cancer risk in veteran men undergoing prostate biopsy. Cancer Epidemiol 2019; 62: 101578.

8. Furue H, Matsuo K, Kumimoto H, et al. Decreased risk of colorectal cancer with the high natural killer cell activity NKG2D genotype in Japanese. Carcinogenesis 2008; 29: 316-20.

9. Imai K, Matsuyama S, Miyake S, Suga K, Nakachi K. Natural cytotoxic activity of peripheral-blood lymphocytes and cancer incidence: an 11-year follow-up study of a general population. Lancet 2000; 356: 1795-9.

10. Ishigami S, Natsugoe S, Tokuda K, et al. Prognostic value of intratumoral natural killer cells in gastric carcinoma. Cancer 2000; 88: 577-83.

11. McGilvray RW, Eagle RA, Watson NFS, et al. NKG2D ligand expression in human colorectal cancer reveals associations with prognosis and evidence for immunoediting. Clin Cancer Res 2009; 15: 6993-7002.

12. Hayashi T, Imai K, Morishita Y, Hayashi I, Kusunoki Y, Nakachi K. Identification of the NKG2D haplotypes associated with natural cytotoxic activity of peripheral blood lymphocytes and cancer immunosurveillance. Cancer Res 2006; 66: 563-70.
13. Jung YS, Kwon MJ, Park D II, Sohn C II, Park JH. Association between natural killer cell activity and the risk of colorectal neoplasia. J Gastroenterol Hepatol 2018; 33: 831-6.

14. Jobin G, Rodriguez-Suarez R, Betito K. Association between natural killer cell activity and colorectal cancer in high-risk subjects undergoing colonoscopy. Gastroenterology 2017; 153: 980-7.

15. Amankulov J, Kaidarova D, Zholdybay Z, et al. Colorectal cancer screening with computed tomography colonography: single region experience in Kazakhstan. Clin Endosc 2021. doi: 10.5946/ce.2021.066

16. Pradier A, Papaserafeim M, Li N, et al. Small-molecule immunosuppressive drugs and therapeutic immunoglobulins differentially inhibit NK cell effector functions in vitro. Front Immunol 2019; 10: 556.

17. Ng S, Deng J, Chinnadurai R, Yuan S, Pennati A, Galipeau J. Stimulation of natural killer cell-mediated tumor immunity by an IL15/TGF $\beta$-neutralizing fusion protein. Cancer Res 2016; 76: 5683-95.

18. Burkard M, Leischner C, Lauer UM, Busch C, Venturelli S, Frank J. Dietary flavonoids and modulation of natural killer cells: implications in malignant and viral diseases. J Nutr Biochem 2017; 46: 1-12.

19. Lee SB, Cha J, Kim IK, et al. A high-throughput assay of NK cell activity in whole blood and its clinical application. Biochem Biophys Res Commun 2014; 445: 584-90.

20. Pickhardt PJ. Screening CT colonography: how I do it. Am J Roentgenol 2007; 189: 290-8.

21. Pooler BD, Kim DH, Lam VP, Burnside ES, Pickhardt PJ. CT Colonography Reporting and Data System (C-RADS): benchmark values from a clinical screening program. Am J Roentgenol 2014; 202: 1232-7.

22. Stoop EM, de Haan MC, de Wijkerslooth TR, et al. Participation and yield of colonoscopy versus non-cathartic CT colonography in population-based screening for colorectal cancer: a randomised controlled trial. Lancet Oncol 2012; 13: 55-64.

23. Tallerico R, Todaro M, Di Franco S, et al. Human NK cells selective targeting of colon cancer-initiating cells: a role for natural cytotoxicity receptors and MHC class I molecules. J Immunol 2013; 190: 2381-90.

24. Halama N, Braun M, Kahlert C, et al. Natural killer cells are scarce in colorectal carcinoma tissue despite high levels of chemokines and cytokines. Clin Cancer Res 2011; 17: 678-89.

25. Johnson CM, Wei C, Ensor JE, et al. Meta-Analyses of colorectal cancer risk factors. Cancer Causes Control 2013; 24: 1207-22.

26. Zhou XY, Yan L, Wang LL, Wang J. Association between physical activity and colorectal cancer risk and prognosis: a meta-analysis. Cancer Treat Res Commun 2016; 9: 62-9.

27. Bigley AB, Spielmann G, Lavoy ECP, Simpson RJ. Can exercise-related improvements in immunity influence cancer prevention and prognosis in the elderly? Maturitas 2013; 76: 51-6.

28. Legaz I, Bolarín JM, Navarro E, et al. KIR2DL2/S2 and KIR2DS5 in alcoholic cirrhotic patients undergoing liver transplantation. Arch Med Sci 2021; 17: 764-74.

29. Zholdybay Z, Amankulov J, Abdrasilova J, Karimbayeva A, Issamatov B, Sadibekova A. Epidemiology of colorectal cancer in Kazakhstan. Sci J Med Vestnik KazNMU 2017; 1: $162-8$. 